\title{
Factors associated with venous thromboembolic events following ICU admission in patients undergoing spinal surgery: an analysis of 1269 consecutive patients
}

\author{
Presented at the 2018 AANS/CNS Joint Section on Disorders of the Spine and Peripheral Nerves \\ Michael Brendan Cloney, MD, MPH, Jack Goergen, BS, Benjamin S. Hopkins, BS, \\ Ekamjeet Singh Dhillon, MD, and Nader S. Dahdaleh, MD
}

Department of Neurological Surgery, Northwestern University Feinberg School of Medicine, Chicago, Illinois

\begin{abstract}
OBJECTIVE Venous thromboembolic events (VTEs) are a common cause of morbidity and mortality after spine surgery. Patients admitted to the intensive care unit (ICU) following spine surgery exhibit high-risk clinical characteristics.

METHODS The authors retrospectively analyzed 1269 ICU patients who had undergone spine surgery between January 1, 2009, and May 31, 2015. Relevant demographic, procedural, and outcome variables were collected.

RESULTS Patients admitted to the ICU postoperatively had a postoperative VTE rate of $10.2 \%$, compared to $2.5 \%$ among all spine surgery patients during the study period. ICU patients had a higher comorbid disease burden (odds ratio $[O R] 1.45, p<0.001)$, and were more likely to have a history of a bleeding disorder $(2.60 \%$ vs $0.46 \%, O R 2.85, p$ $=0.028$ ), receive a transfusion (OR 4.81, $p<0.001$ ), have a fracture repaired (OR 4.30, $p<0.001$ ), have an estimated blood loss $>500 \mathrm{ml}$ (OR 1.95, $p=0.009$ ), have an osteotomy (OR 20.47, $p=0.006)$, or have a corpectomy (OR 3.48, $p=$ 0.007 ) than patients not admitted to the ICU. There was a significant difference in time to VTE between patients undergoing osteotomy and patients undergoing scoliosis corrections without osteotomy $(p=0.0431)$, patients with fractures $(p=0.0113)$, and patients undergoing fusions for indications other than scoliosis or fracture $(p=0.0056)$. Patients who developed a deep vein thrombosis (DVT) during their ICU stay were more likely to have received a prophylactic inferior vena cava filter placement (OR 8.98, $p<0.001)$, have undergone an interbody fusion procedure (OR 2.38, $p=0.037)$, have a history of DVT (OR $3.25, p<0.001)$, and have shorter surgery times (OR $0.30, p=0.002)$. Patients who developed a pulmonary embolism (PE) during the ICU stay were more likely to have a history of PE $(\mathrm{OR} 12.68 p=0.015)$, history of DVT (OR 5.11, $p=0.042$ ), fracture diagnosis (OR 7.02, $p=0.040)$, and diagnosis of scoliosis (OR 7.78, $p=$ 0.024). Patients with higher BMls (OR $0.85, p=0.036$ ) and those who received anticoagulation treatment (OR $0.16, p=$ 0.031) were less likely to develop a PE during their ICU stay.

CONCLUSIONS Patients admitted to the ICU following spine surgery have a higher rate of VTE than non-ICU patients Time to VTE varied by pathology. Factors independently associated with VTE in the ICU are distinct from factors otherwise associated with VTE. Some factors are independently associated with VTE throughout the 30-day postoperative period, while others are associated with VTE specifically during the initial ICU stay or after leaving the ICU.

https://thejns.org/doi/abs/10.3171/2018.5.SPINE171027
\end{abstract}

KEYWORDS spine surgery; intensive care unit; venous thromboembolism; pulmonary embolism; deep venous thrombosis; vascular disorders

$\mathrm{V}$ ENOus thromboembolic events (VTEs), which include pulmonary embolisms (PEs) and deep vein thrombosis (DVT), are a common cause of morbidity and mortality after spine surgery.1,13,17 Rates vary across patient populations, with one recent systematic review noting a DVT rate of $2.7 \%$ and PE rate of $2.1 \%$ across the broader spine surgery population, but with rates as high as $31 \%$ in high-risk patient populations. ${ }^{16}$

Patients admitted to the intensive care unit (ICU) following spine surgery are typically in poorer health and have a higher propensity for comorbid disease, placing them at a higher risk of developing VTEs and other com-

ABBREVIATIONS CPT = Current Procedural Terminology; DVT = deep vein thrombosis; EBL = estimated blood loss; ICU = intensive care unit; IVC = inferior vena cava; $\mathrm{PE}=$ pulmonary embolism; $\mathrm{OR}=$ odds ratio; $\mathrm{VTE}=$ venous thromboembolic event.

SUBMITTED January 20, 2018. ACCEPTED May 25, 2018.

INCLUDE WHEN CITING Published online October 12, 2018; DOI: 10.3171/2018.5.SPINE171027. 
plications. ${ }^{23,24}$ Roughly $65 \%$ of ICU patients have comorbid medical diagnoses, ${ }^{23,25}$ making VTEs particularly dangerous in this patient subset. ${ }^{10,31}$ Further insight is still needed to guide treatment and prophylaxis in unique highrisk populations such as ICU patients. ${ }^{19}$

The goal of this study was to identify risk factors for VTEs following spine surgery in a high-risk subset of patients who were admitted to the ICU postoperatively. While considerable research has gone into investigating postsurgical VTEs in the general population, the literature is limited for this high-risk subset of patients. In the current study, we examined clinical and demographic characteristics of patients who were selected for ICU admission after undergoing spine surgery, and identified factors independently associated with VTE in this unique patient population both during their ICU admission and at other time points during their hospitalization.

\section{Methods \\ Data Source}

All spine patients at Northwestern Memorial Hospital were identified between January 1, 2009, and May 31, 2015, using the Northwestern University Electronic Data Warehouse, a clinical data repository funded in conjunction with Northwestern Memorial Hospital serving the purpose of large clinical data storage. Current Procedural Terminology (CPT) codes were used to find and identify all primary spine surgeries. In patients with multiple operations requiring different admissions during this timeframe, each operation was analyzed separately. All patients undergoing minor spinal procedures or secondary procedures were excluded from analysis (including electrode placements, instrumentation removals, operations for wound dehiscence, and hematoma evacuations). For each study subject, data were collected about the patient, procedure, and postoperative management. The IRB of Northwestern University approved this study.

\section{Patient Data}

We collected the following patient data: age at surgery, sex, BMI, history of bleeding disorders, smoking status (never, ever, and current), history of VTEs, and number of comorbidities present (including hypertension, cardiac, renal, pulmonary, and endocrine disease) as identified by ICD-9 codes.

\section{Procedural Data}

We collected the following data about the procedures performed: site of surgery (cervical, thoracic, lumbar, unspecified), length of surgery, estimated blood loss (EBL; in $\mathrm{ml}$ ), use of transfusion, length of ICU stay (hours), and length of hospitalization (days). We also collected data on whether the following were part of the patient's presenting pathology or the procedure performed: fusion, interbody fusion, laminectomy, corpectomy, osteotomy, kyphoplasty, minimally invasive surgery, cyst or abscesses, fractures, tumors, stenosis, scoliosis, prior inferior vena cava (IVC) filter placement, length of stay, and use of chemoprophylactic anticoagulation.

\section{Outcomes Data}

We collected data on the occurrence of VTE complications within 30 days after surgery, defined as either DVT or PE. We collected data on the use of chemoprophylactic anticoagulation in our patients. Given recent data showing that spine surgeons using chemoprophylactic anticoagulation do so in the first few days following surgery, ${ }^{16}$ we defined chemoprophylaxis as anticoagulation given from 1 day prior to 3 days after the operation. Patients who received anticoagulation outside this window were assumed to be receiving it therapeutically and were not included in the chemoprophylactic group.

\section{Statistical Methods}

Microsoft Excel 2015 was used to manage and collect data. Statistical analysis was performed using Stata (version 14.0, StataCorp). Parametric data were presented as the mean \pm standard deviation and compared using 2-tailed t-tests. Nonparametric data were compared using the Mann-Whitney U-test or chi-square test, as appropriate. Regression analysis was performed using stepwise logistic regression. A threshold of $p<0.10$ was used for multivariate inclusion and a $\mathrm{p}$ value $<0.05$ was considered statistically significant.

\section{Results}

\section{Selection for ICU Admission}

We identified 6869 consecutive patients who underwent spine surgery at our institution during the period studied. From them, we identified 1269 patients who were admitted to the ICU postoperatively. Patients in the group admitted to the ICU had longer surgeries (odds ratio [OR] $4.25, \mathrm{p}<0.001$ ), higher BMIs (OR 1.03, $\mathrm{p}=0.027$ ), and longer hospital stays (OR 1.45, $\mathrm{p}<0.001$ ). These patients also had a higher comorbid disease burden (OR 1.45, p < 0.001), higher likelihood of a history of a bleeding disor$\operatorname{der}(2.60 \%$ vs $0.46 \%$, OR $2.85, \mathrm{p}=0.028)$, and were more likely to be male (OR 2.01, p < 0.001). Patients admitted to the ICU were also more likely to receive a transfusion (OR 4.81, $\mathrm{p}<0.001)$, have a fracture repaired (OR 4.29, $\mathrm{p}$ $<0.001)$, have an EBL > $500 \mathrm{ml}(\mathrm{OR} 1.95, \mathrm{p}=0.009)$, have an osteotomy (OR 20.47, $\mathrm{p}=0.006$ ), or have a corpectomy $(\mathrm{OR} 3.48, \mathrm{p}=0.007$; Table 1$)$.

\section{Demographic and Clinical Data}

Among patients selected for ICU admission, the mean patient age was 58.9 years, and the median age was 61 years. Of 1269 patients, 645 were male $(50.83 \%)$. The mean length of hospital stay was 9.25 days, with a median of 7 days. The mean length of ICU stay was 81.0 hours with a median of 48 hours. Our patient cohort included 864 white patients (68.09\%), 139 African Americans (10.95\%), 63 Hispanics (4.96\%), 4 Asian Pacific Islanders (0.32\%), and 199 patients who identified as other (15.68\%). A fusion was performed on 900 patients (70.92\%), an osteotomy was performed on 121 patients (9.54\%), 149 patients (11.74\%) had a scoliosis correction, 133 patients $(10.48 \%)$ had surgery for a fracture, and 88 patients (6.93\%) underwent a corpectomy (Table 2). 
TABLE 1. Characteristics of patients admitted to the ICU compared to patients not admitted, as determined by multivariable logistic regression

\begin{tabular}{lccc}
\hline \multicolumn{1}{c}{ Characteristic } & $p$ Value & OR & $95 \% \mathrm{Cl}$ \\
\hline Surgery $>4$ hrs & $<0.001$ & 4.253512 & $2.985032-6.061028$ \\
\hline Length of stay & $<0.001$ & 1.445626 & $1.355395-1.541863$ \\
\hline Transfusion & $<0.001$ & 4.807072 & $2.946314-7.843001$ \\
\hline Lumbar surgery & $<0.001$ & 0.2856406 & $0.1988776-0.410255$ \\
\hline $\begin{array}{l}\text { Comorbid disease } \\
\text { burden }\end{array}$ & $<0.001$ & 1.450795 & $1.21503-1.732309$ \\
\hline Sex & $<0.001$ & 2.012157 & $1.445438-2.801074$ \\
\hline Fracture & $<0.001$ & 4.294704 & $1.990139-9.267936$ \\
\hline EBL $>500 \mathrm{ml}$ & 0.009 & 1.946183 & $1.185028-3.196234$ \\
\hline Osteotomy & 0.006 & 20.47045 & $2.393249-175.0922$ \\
\hline $\begin{array}{l}\text { History of bleeding } \\
\text { disorder }\end{array}$ & 0.028 & 2.854518 & $1.120385-7.27274$ \\
\hline Corpectomy & 0.007 & 3.481403 & $1.39527-8.686608$ \\
\hline BMl & 0.027 & 1.026497 & $1.003012-1.05053$ \\
\hline
\end{tabular}

\section{VTE Rates and Use of Chemoprophylactic Anticoagulation}

Among the 1269 ICU patients included in our study, 130 experienced VTEs (10.2\%), including 110 DVTs (8.7\%), and 35 PEs (2.8\%). The median time of VTE development was 4.48 days overall, and 4.41, 4.90, 4.01, and 8.03 days for patients undergoing interbody fusion, laminotomies, foraminotomies, and osteotomies, respectively. Of these 130 patients, 59 experienced VTEs while in the ICU, including 18 PEs and 53 DVTs. Of the patients admitted to the ICU following spine surgery, 59.18\% ( $\mathrm{n}=$ 751) received anticoagulant therapy for chemoprophylaxis, much greater than the $22.80 \%(n=1276)$ of non-ICU admits who received chemoprophylaxis. Chemoprophylactic anticoagulant choice among the subset of ICU patients is shown in Table 3. Among ICU patients, increasing age was independently associated with selection for chemoprophylaxis $(\mathrm{OR} 1.02, \mathrm{p}=0.012)$. There was a trend toward a difference in time to VTEs when patients were stratified by pathology and procedure $(\mathrm{p}=0.064)$. On direct comparison, there was a significant difference in time to VTEs between patients undergoing osteotomy and those undergoing scoliosis corrections without osteotomy, patients with fractures, and patients undergoing fusions for indications other than scoliosis or fracture (Table 4, Fig. 1). There were no differences in time to VTEs among the other groups. Despite prophylactic use, treatment failure occurred in 58 of 751 patients $(7.7 \%)$ who were receiving chemoprophylaxis (11 PEs, 47 DVTs) with events occurring a median of 6.12 days postoperatively.

\section{Factors Independently Associated With DVT}

Among patients selected for ICU admission, those who developed a DVT within 30 days postoperatively were more like to have a history of DVT (OR 3.25, p < 0.001), have a longer length of hospital stay (OR 1.05, $\mathrm{p}=0.002)$, have received a prophylactic IVC filter placement (OR $3.41, \mathrm{p}<0.001)$, and have undergone an interbody fusion
TABLE 2. Demographic and clinical characteristics of 1269 consecutive patients admitted to the ICU after undergoing spine surgery

\begin{tabular}{lc}
\hline \multicolumn{1}{c}{ Characteristic } & Value (\%) \\
\hline Mean age, yrs & 58.94 \\
\hline Median age, yrs & 61 \\
\hline Race & $864(68)$ \\
\hline White & $139(11)$ \\
\hline African American & $63(5)$ \\
\hline Hispanic & $4(0.3)$ \\
\hline Pacific Islander & $199(16)$ \\
\hline Other & 9.25 \\
\hline Mean length of hospital stay, days & 7 \\
\hline Median length of hospital stay, days & 81.03 \\
\hline Mean length of ICU stay, hrs & 48 \\
\hline Median length of ICU stay, hrs & \\
\hline Surgical characteristics & $121(10)$ \\
\hline Osteotomy & $900(71)$ \\
\hline Fusion & $149(12)$ \\
\hline Scoliosis correction & $88(7)$ \\
\hline Corpectomy & $133(10)$ \\
\hline Fracture correction & $163(13)$ \\
\hline Laminectomy w/o fusion & $347(27)$ \\
\hline Cervical region & $297(23)$ \\
\hline Thoracic region & $595(47)$ \\
\hline Lumbar region &
\end{tabular}

procedure $(\mathrm{OR} 2.38, \mathrm{p}=0.037)$ compared to those who did not (Table 5).

Patients who developed a DVT during their ICU stay were more likely to have received a prophylactic IVC filter placement (OR 8.98, p < 0.001), have undergone an interbody fusion procedure (OR 2.38, $\mathrm{p}=0.037$ ), have a history of DVT (OR 3.25, p < 0.001), and have shorter surgery times (OR 0.30, $\mathrm{p}=0.002$ ) compared to those who did not develop a DVT during their hospital stay. Notably, there was a trend toward significance in patients with a fracture diagnosis (OR 3.09, $\mathrm{p}=0.051)$ and with a history of PE (OR 3.54, $\mathrm{p}=0.066)$.

TABLE 3. Anticoagulation choice after spine surgery $(n=849)$

\begin{tabular}{lccc}
\hline & & \multicolumn{2}{c}{ Start Time After Surgery (days) } \\
\cline { 3 - 4 } Anticoagulant & Percentage & Mean & Median \\
\hline Warfarin & 2.83 & 9.85 & 7.68 \\
\hline Heparin & 31.57 & 1.94 & 1.67 \\
\hline Enoxaparin & 23.32 & 1.98 & 1.11 \\
\hline Dalteparin & 41.58 & 1.93 & 1.86 \\
\hline Foundaparinux & 0.71 & 4.00 & 3.94 \\
\hline
\end{tabular}

Choice of anticoagulation for all 751 patients selected for ICU admission who received chemoprophylaxis. The total number of anticoagulants $(n=849)$ is greater than the total number of patients who received chemoprophylaxis ( $\mathrm{n}$ $=751$ ) because some patients required multiple different chemoprophylactic agents during their ICU stay. 
TABLE 4. Median time to VTE, stratified by type of pathology or surgical intervention

\begin{tabular}{lcc}
\hline \multicolumn{1}{c}{ Group } & Median Days to VTE & p Value \\
\hline Fusion & 3.97 & 0.0056 \\
\hline Fracture & 4.28 & 0.0113 \\
\hline Scoliosis & 5.95 & 0.0431 \\
\hline Laminectomy & 6.08 & 0.2371 \\
\hline Osteotomy & 8.16 & - \\
\hline
\end{tabular}

The $p$ values in the above table represent $p$ values associated with direct comparisons between a given pathology or intervention, and the reference group (the osteotomy group). There was a trend toward an overall difference in time to VTEs $(p=0.064)$.

Patients who developed a DVT after their ICU stay were more likely to have a history of DVT (OR 6.45, p < 0.001 ), an interbody fusion (OR 2.64, $\mathrm{p}=0.047$ ), or osteotomy procedure (OR 3.14, $\mathrm{p}=0.045$ ) compared to those who did not develop a DVT after their ICU stay. Patients who underwent a fusion other than an interbody fusion were less likely to develop a DVT during their ICU stay (OR 0.36, $\mathrm{p}=0.017)$ than other ICU patients.

\section{Factors Independently Associated With PE}

Among patients selected for ICU admission, those developing $\mathrm{PE}$ within 30 days postoperatively were more likely to have a history of PE (OR 15.65, p < 0.001), more likely to have undergone a surgery longer than 4 hours (OR $3.84, p=0.047)$, and less likely to be receiving chemoprophylaxis (OR 0.34, $\mathrm{p}=0.021$ ) compared to those who did not develop a PE during their hospital stay (Table 6).

Patients who developed a PE during the ICU stay were more likely to have a history of PE (OR 12.68, $\mathrm{p}=0.015$ ), history of DVT (OR 5.11, $\mathrm{p}=0.042$ ), fracture diagnosis $(\mathrm{OR} 7.02, \mathrm{p}=0.040)$, and diagnosis of scoliosis (OR 7.78, $\mathrm{p}=0.024)$ compared to those who did not develop a PE during their ICU stay. Interestingly, patients with higher BMIs were less likely to develop a PE during their ICU stay $(\mathrm{OR} 0.85, \mathrm{p}=0.036)$. Patients receiving anticoagulant therapy for chemoprophylaxis were less likely to develop a PE during their ICU stay compared to those who did not (OR 0.16, $\mathrm{p}=0.031$ ).

Patients who developed a PE after their ICU stay were more likely to have a history of PE (OR 37.48, p < 0.001), or to have undergone a laminectomy (OR 3.81, $\mathrm{p}=0.041$ ), and were less likely to be men (OR 0.12, p = 0.006), compared to those who did not develop a PE following their ICU stay. Patients receiving a transfusion were less likely to develop a PE following their ICU stay compared to those who did not receive any blood products (OR 0.16, $\mathrm{p}=0.043)$.

\section{Discussion}

Venous thromboembolism following spine surgery is a common cause of significant morbidity and mortality. ${ }^{14,16,26}$ Much of the current literature focuses on the epidemiology of VTE following specific surgical procedures, although specific subgroups of patients within such cohorts are likely at higher risk of VTE than others., ${ }^{2,3}$ Patients admitted to the ICU following spine surgery are typically in poorer health and have a higher propensity for comorbid disease, placing them at a higher risk of developing VTEs and other complications. ${ }^{23,24}$ Further insight is still needed to guide treatment and prophylaxis in unique, high-risk populations such as ICU patients..$^{19}$ In this study we identi-
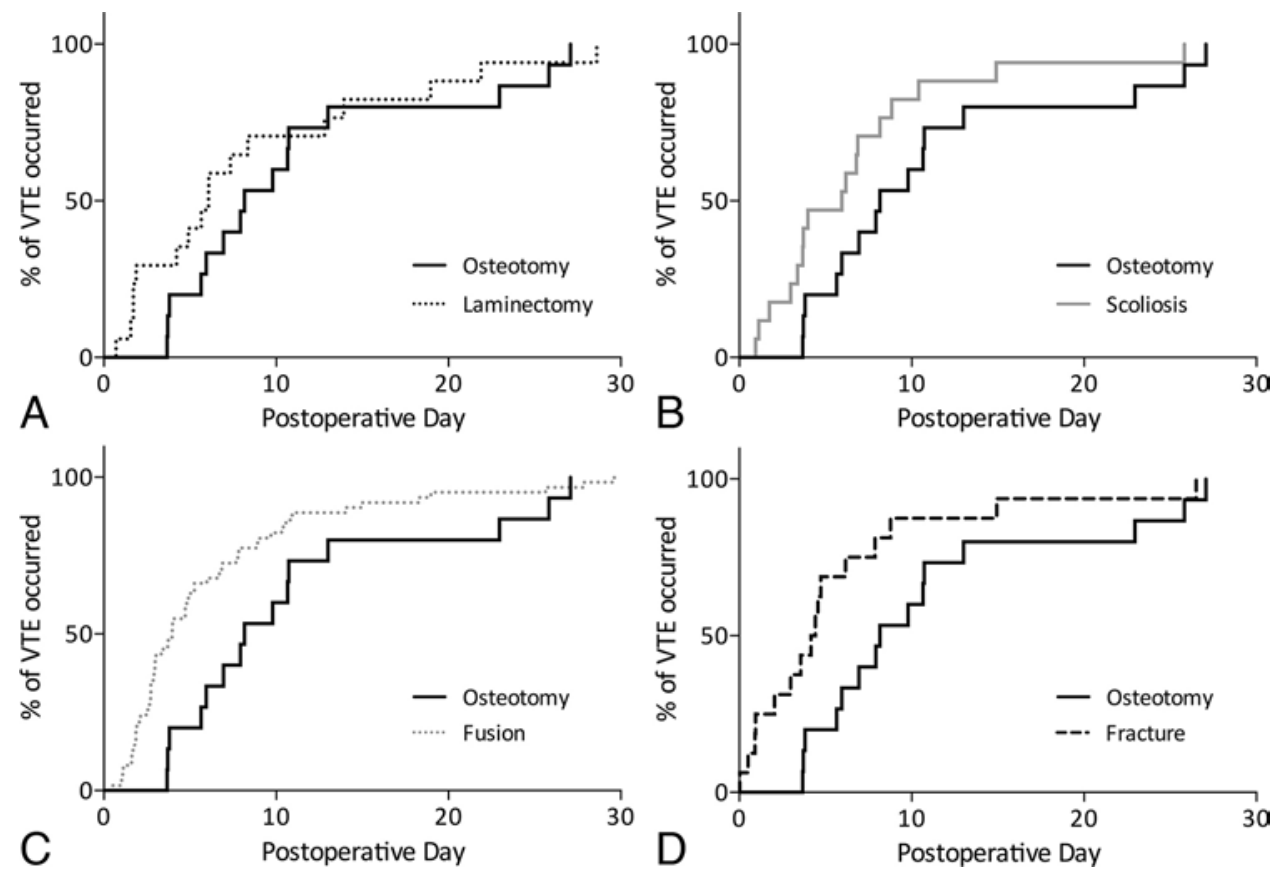

FIG. 1. Difference in time to VTE between patients based on procedure characteristics. A significant difference was found between patients undergoing osteotomy versus patients undergoing scoliosis corrections without osteotomy $(p=0.0431)$, versus patients with fractures $(p=0.0113)$, and versus patients undergoing fusions for indications other than scoliosis or fracture $(p=0.0056)$. 
TABLE 5. Factors independently associated with DVT among 1269 consecutive patients admitted to the ICU after undergoing spine surgery

\begin{tabular}{crcc}
\hline \multicolumn{1}{c}{ Factor } & p Value & \multicolumn{1}{c}{ OR } & $95 \% \mathrm{Cl}$ \\
\hline $\begin{array}{c}\text { DVT w/in } 30 \text { days } \\
\text { postop }\end{array}$ & & & \\
\hline IVC filter placed & $<0.001$ & 3.412723 & $1.808512-6.439924$ \\
\hline History of DVT & $<0.001$ & 3.249682 & $1.70479-6.194565$ \\
\hline Length of stay & 0.002 & 1.053398 & $1.018483-1.08951$ \\
\hline Interbody fusion & 0.037 & 2.380605 & $1.051834-5.387999$ \\
\hline $\begin{array}{c}\text { DVT during postop } \\
\text { ICU stay }\end{array}$ & & & \\
\hline IVC filter placed & $<0.001$ & 8.98246 & $3.515054-22.95401$ \\
\hline Interbody fusion & 0.037 & 2.380605 & - \\
\hline History of DVT & $<0.001$ & 3.249682 & - \\
\hline Surgery $>4$ hrs & 0.015 & 0.3014445 & $0.1151172-0.7893586$ \\
\hline History of PE & 0.066 & 3.542863 & - \\
\hline Fracture & 0.051 & 3.090874 & \\
\hline DVT after postop ICU & & & - \\
stay & & & \\
\hline History of DVT & $<0.001$ & 6.427699 & $2.811318-14.69606$ \\
\hline Foraminotomy & 0.042 & 4.176589 & $1.051321-16.59237$ \\
\hline Fusion & 0.017 & 0.3567093 & $0.1533802-0.8295825$ \\
\hline Interbody fusion & 0.047 & 2.641959 & $1.013242-6.888731$ \\
\hline Osteotomy & 0.045 & 3.139169 & $1.025886-9.605729$ \\
\hline
\end{tabular}

Separate multivariable logistic regression analyses were performed to identify variables associated with DVT during the postoperative ICU stay, DVT after leaving the ICU, and DVT at any point within the first 30 postoperative days.

fied factors that were independently associated with being selected for ICU admission after surgery, as well as factors independently associated with VTEs in this unique patient subgroup, both within 30 postoperative days and their postoperative ICU stay specifically.

In our study, we found that patients selected for ICU admission had a higher BMI, were more likely to have a history of a bleeding disorder, and had a higher comorbid disease burden. They also underwent longer surgeries, were more likely to receive transfusions, had more blood loss during surgery, and had other similar perioperative differences. These findings are consistent with previous literature on ICU patients that shows similar differences between ICU and non-ICU patients. ${ }^{4,7,8,12}$

In our high-risk patient population, we found that there was an overall VTE rate of $10.2 \%$, compared to a rate of $2.5 \%$ for all patients presenting to our institution during the same time period. ${ }^{11}$ Our overall $2.5 \%$ rate of VTE is comparable to the rate observed in a recent systematic review of 25 studies. ${ }^{16}$ Existing literature is lacking on rates of VTE after spine surgery among patients requiring postoperative ICU care, but their higher rate of VTE is expected given their high-risk clinical characteristics.

Our findings suggest that the factors associated with the development of DVT in the ICU are different than the factors associated with DVT at other times during the 30-day postoperative period. Having a history of DVT or undergoing an interbody fusion was associated with
TABLE 6. Factors independently associated with PE among 1269 consecutive patients admitted to the ICU after undergoing spine surgery

\begin{tabular}{cccc}
\hline \multicolumn{1}{c}{ Factor } & p Value & OR & $95 \% \mathrm{Cl}$ \\
\hline $\begin{array}{c}\text { PE w/in } 30 \text { days } \\
\text { postop }\end{array}$ & & & \\
\hline History of PE & $<0.001$ & 15.64945 & $4.431427-55.26557$ \\
\hline $\begin{array}{c}\text { Chemoprophy- } \\
\text { laxis }\end{array}$ & 0.021 & 0.3363742 & $0.1334331-0.8479725$ \\
\hline Surgery $>4$ hrs & 0.047 & 3.841612 & $1.015781-14.5287$ \\
\hline PE in ICU & & & \\
\hline History of PE & 0.015 & 12.68318 & $1.62243-99.1495$ \\
\hline Fracture & 0.04 & 7.017144 & $1.08992-45.1779$ \\
\hline BMI & 0.036 & 0.8490821 & $0.7285448-0.9895622$ \\
\hline History of DVT & 0.042 & 5.107954 & $1.056872-24.68718$ \\
\hline Chemoprophylaxis & 0.031 & 0.1623893 & $0.0310727-0.8486636$ \\
\hline Scoliosis & 0.024 & 7.782698 & $1.31439-46.08252$ \\
\hline PE after ICU & & & \\
\hline History of PE & $<0.001$ & 37.4806 & $6.068861-231.476$ \\
\hline Sex & 0.006 & 0.1239585 & $0.0278022-0.5526794$ \\
\hline Laminectomy & 0.041 & 3.807712 & $1.058083-13.70277$ \\
\hline Transfusion & 0.043 & 0.1556681 & $0.0255944-0.9467926$ \\
\hline
\end{tabular}

Chemoprophylaxis $=$ chemoprophylactic anticoagulation .

Separate multivariable logistic regression analyses were performed to identify variables associated with PE during the postoperative ICU stay, PE after leaving the ICU, and $\mathrm{PE}$ at any point within the first 30 postoperative days.

DVT both during the ICU stay and after leaving the ICU, which is consistent with existing literature showing that these are DVT risk factors. ${ }^{5,21,30}$ Surgery for a spinal fracture approached significance in association with developing DVT in the ICU, while patients undergoing osteotomy were more likely to develop DVT after leaving the ICU. This is consistent with our finding that there was a significant difference in time to VTE between patients with fractures and patients who underwent osteotomy. Moreover, existing literature, including a meta-analysis encompassing 29 studies, has shown that VTE risk varies depending on the procedure performed and whether the procedure is performed for deformity or a degenerative condition..$^{9,26}$ Further studies are required to determine the driver of this difference in the timing of VTE between patient populations.

Different factors were independently associated with PE during ICU stay and PE at any other time during the 30-day postoperative period. Consistent with the current literature on VTE risk factors, $5,21,30$ a history of VTE or $\mathrm{PE}$ was associated with PE, either during the ICU stay or after leaving the ICU. Undergoing surgery for a fracture or for scoliosis were both associated with PE during the ICU stay, while laminectomy was associated with PE after leaving the ICU. This may reflect the differences in the baseline risk for PE between these patients, as well as the effects of each surgery. The endothelial disruption associated with a scoliosis correction or fractures may cause an acutely hypercoaguable state in such patients, making them more likely to develop PE in the immediate postop- 
erative period, during the ICU stay. In contrast, patients requiring laminectomy have elsewhere been described to be at decreased risk of PE compared to patients with deformity. ${ }^{9}$ This decreased risk may be attributable to the fact that laminectomies are on average less complicated procedures than various scoliosis correction procedures and fracture correction and are therefore less likely to cause extensive endothelial damage in comparison. However, risk factors for VTEs in patients who underwent laminectomy, such as age and comorbid disease burden, would likely remain constant throughout a patient's hospital stay and may contribute to their propensity for out-of-ICU PE events.

Similarly, female sex is an unchanging risk factor that was associated with $\mathrm{PE}$ during the 30 -day postoperative period, which is consistent with prior spine surgery literature showing an increased VTE risk in women. ${ }^{20}$ Interestingly, in contrast with prior studies showing that a higher BMI is associated with VTEs, ${ }^{28,30}$ we found that patients with a higher BMI were less likely to have a PE while in the ICU. It is unclear to the authors why such an association was found or what specifically may be driving this finding. It is possible that patients with an increased BMI are more likely to undergo more liberal precautionary management or prophylaxis so as to prevent unnecessary complications. Similarly, patients with higher BMIs may experience extended recovery times, longer immobilization, and more perioperative complications, predisposing them to prolonged hospital courses outside the ICU during which VTEs may occur. However, it is difficult to draw conclusions without further investigation.

Importantly, chemoprophylaxis was associated with a significant decrease in PE in the ICU setting. It is well established that anticoagulation decreases VTEs. ${ }^{5,13,18}$ However, there is controversy about how to balance the benefits of chemoprophylaxis with the risk of bleeding complications in the immediate postoperative period, and there is significant variability in the use of prophylactic anticoagulation after spine surgery., ${ }^{6,14-16}$ Given the significant morbidity and mortality associated with PE, chemoprophylactic anticoagulation may be beneficial for spine surgery patients selected for ICU admission.

There are multiple limitations to our study. First, this is a retrospective study and it is therefore prone to information bias. Detection bias could have impacted the number of thromboembolisms discovered as there is no universal DVT screening protocol at our institution and the decision to perform screening ultrasounds is dependent on individual provider preference. The patients in the ICU were likely under intense observation and suspicion from their providers due to their elevated risk, potentially affecting the number of VTEs discovered compared to the non-ICU population. Similarly, as the current study was performed at a single institution only, it remains to be seen whether or not found results will be generalizable across various unique institutions across the country. Despite these limitations, our study provides much needed insight into a high-risk patient population for which the existing literature is limited.

Prior studies on patients undergoing spine surgeries have also focused on the rate of VTE and its risk factors. ${ }^{2,5,13,22,27,29}$ However, these analyses have either focused on specific procedures or have investigated specific patient populations other than patients in the ICU setting. Our analysis adds to the existing literature by examining risk factors for VTEs among postoperative spine patients who are admitted to the ICU, and by distinguishing between factors independently associated with VTE during ICU stay as opposed to the remainder of a 30-day postoperative period. By examining this specific population, our study provides insight for a high-risk group that has not been a focus of previous spine studies.

\section{Conclusions}

Patients admitted to the ICU following spine surgery have a higher rate of VTEs than non-ICU patients. Patients admitted to the ICU following spine surgery are typically in poorer health, and are undergoing high-risk surgeries. Time to VTE varied by pathology. Factors independently associated with VTEs in the ICU are distinct from factors otherwise associated with VTE. Some factors are independently associated with VTEs throughout the 30day postoperative period, while others are associated with VTE specifically during the initial ICU stay or after leaving the ICU.

\section{References}

1. Agnelli G, Buller HR, Cohen A, Curto M, Gallus AS, Johnson M, et al: Oral apixaban for the treatment of acute venous thromboembolism. N Engl J Med 369:799-808, 2013

2. Akeda K, Matsunaga H, Imanishi T, Hasegawa M, Sakakibara T, Kasai Y, et al: Prevalence and countermeasures for venous thromboembolic diseases associated with spinal surgery: a follow-up study of an institutional protocol in 209 patients. Spine (Phila Pa 1976) 39:791-797, 2014

3. Al-Dujaili TM, Majer CN, Madhoun TE, Kassis SZ, Saleh AA: Deep venous thrombosis in spine surgery patients: incidence and hematoma formation. Int Surg 97:150-154, 2012

4. Angus DC, Carlet J: Surviving intensive care: a report from the 2002 Brussels Roundtable. Intensive Care Med 29:368377,2003

5. Brambilla S, Ruosi C, La Maida GA, Caserta S: Prevention of venous thromboembolism in spinal surgery. Eur Spine J 13:1-8, 2004

6. Bryson DJ, Uzoigwe CE, Braybrooke J: Thromboprophylaxis in spinal surgery: a survey. J Orthop Surg Res 7:14, 2012

7. Buckley TA, Cheng AY, Gomersall CD: Quality of life in long-term survivors of intensive care. Ann Acad Med Singapore 30:287-292, 2001

8. Chaboyer W, Elliott D: Health-related quality of life of ICU survivors: review of the literature. Intensive Crit Care Nurs 16:88-97, 2000

9. Cheng JS, Arnold PM, Anderson PA, Fischer D, Dettori JR: Anticoagulation risk in spine surgery. Spine (Phila Pa 1976) 35 (9 Suppl):S117-S124, 2010

10. Cook D, Meade M, Guyatt G, Walter S, Heels-Ansdell D, Warkentin TE, et al: Dalteparin versus unfractionated heparin in critically ill patients. N Engl J Med 364:1305-1314, 2011

11. Dhillon ES, Khanna R, Cloney M, Roberts H, Cybulski GR, Koski TR, et al: Timing and risks of chemoprophylaxis after spinal surgery: a single-center experience with 6869 consecutive patients. J Neurosurg Spine 27:681-693, 2017

12. Dowdy DW, Eid MP, Sedrakyan A, Mendez-Tellez PA, Pronovost PJ, Herridge MS, et al: Quality of life in adult survivors of critical illness: a systematic review of the literature. Intensive Care Med 31:611-620, 2005 
13. Epstein NE: A review of the risks and benefits of differing prophylaxis regimens for the treatment of deep venous thrombosis and pulmonary embolism in neurosurgery. Surg Neurol 64:295-302, 2005

14. Geerts WH, Bergqvist D, Pineo GF, Heit JA, Samama CM, Lassen MR, et al: Prevention of venous thromboembolism: American College of Chest Physicians Evidence-Based Clinical Practice Guidelines (8th Edition). Chest 133:381S-453S, 2008

15. Glotzbecker MP, Bono CM, Harris MB, Brick G, Heary RF, Wood KB: Surgeon practices regarding postoperative thromboembolic prophylaxis after high-risk spinal surgery. Spine (Phila Pa 1976) 33:2915-2921, 2008

16. Glotzbecker MP, Bono CM, Wood KB, Harris MB: Thromboembolic disease in spinal surgery: a systematic review. Spine (Phila Pa 1976) 34:291-303, 2009

17. Goldhaber SZ, Bounameaux H: Pulmonary embolism and deep vein thrombosis. Lancet 379:1835-1846, 2012

18. Hill J, Treasure T: Reducing the risk of venous thromboembolism (deep vein thrombosis and pulmonary embolism) in patients admitted to hospital: summary of the NICE guideline. Heart 96:879-882, 2010

19. Johanson NA, Lachiewicz PF, Lieberman JR, Lotke PA, Parvizi J, Pellegrini V, et al: American Academy of Orthopaedic Surgeons clinical practice guideline on: prevention of symptomatic pulmonary embolism in patients undergoing total hip or knee arthroplasty. J Bone Joint Surg Am 91:1756-1757, 2009

20. Jones T, Ugalde V, Franks P, Zhou H, White RH: Venous thromboembolism after spinal cord injury: incidence, time course, and associated risk factors in 16,240 adults and children. Arch Phys Med Rehabil 86:2240-2247, 2005

21. McClendon J Jr, O'Shaughnessy BA, Smith TR, Sugrue PA, Halpin RJ, Morasch M, et al: Comprehensive assessment of prophylactic preoperative inferior vena cava filters for major spinal reconstruction in adults. Spine (Phila Pa 1976) 37:1122-1129, 2012

22. McClendon J Jr, Smith TR, O'Shaughnessy BA, Sugrue PA, Thompson SE, Koski TR: Time to event analysis for the development of venous thromboembolism after spinal fusion $\geq$ 5 levels. World Neurosurg 84:826-833, 2015

23. Metnitz PG, Moreno RP, Almeida E, Jordan B, Bauer P, Campos RA, et al: SAPS 3-from evaluation of the patient to evaluation of the intensive care unit. Part 1: objectives, methods and cohort description. Intensive Care Med 31:13361344,2005

24. Moreno R, Miranda DR, Matos R, Fevereiro T: Mortality after discharge from intensive care: the impact of organ system failure and nursing workload use at discharge. Intensive Care Med 27:999-1004, 2001

25. Moreno R, Morais P: Outcome prediction in intensive care: results of a prospective, multicentre, Portuguese study. Intensive Care Med 23:177-186, 1997

26. Oda T, Fuji T, Kato Y, Fujita S, Kanemitsu N: Deep venous thrombosis after posterior spinal surgery. Spine (Phila Pa 1976) 25:2962-2967, 2000

27. Sansone JM, del Rio AM, Anderson PA: The prevalence of and specific risk factors for venous thromboembolic disease following elective spine surgery. J Bone Joint Surg Am 92:304-313, 2010

28. Schoenfeld AJ, Herzog JP, Dunn JC, Bader JO, Belmont PJ $\mathrm{Jr}$ : Patient-based and surgical characteristics associated with the acute development of deep venous thrombosis and pulmonary embolism after spine surgery. Spine (Phila Pa 1976) 38:1892-1898, 2013

29. Schulte LM, O'Brien JR, Bean MC, Pierce TP, Yu WD, Meals C: Deep vein thrombosis and pulmonary embolism after spine surgery: incidence and patient risk factors. Am J Orthop 42:267-270, 2013

30. Wang TY, Sakamoto JT, Nayar G, Suresh V, Loriaux DB, Desai R, et al: Independent predictors of 30-day perioperative deep vein thrombosis in 1346 consecutive patients after spine surgery. World Neurosurg 84:1605-1612, 2015

31. Yang JC: Prevention and treatment of deep vein thrombosis and pulmonary embolism in critically ill patients. Crit Care Nurs Q 28:72-79, 2005

\section{Disclosures}

Dr. Dahdaleh reports being a consultant for DePuy Synthes.

\section{Author Contributions}

Conception and design: Cloney. Acquisition of data: Cloney, Hopkins, Dhillon. Analysis and interpretation of data: Cloney. Drafting the article: Goergen, Hopkins. Critically revising the article: Cloney, Dhillon. Reviewed submitted version of manuscript: Dahdaleh. Statistical analysis: Cloney.

\section{Supplemental Information}

\section{Previous Presentations}

This paper was presented as an abstract for an oral presentation at the 2018 AANS/CNS Joint Spine Section on Disorders of the Spine and Peripheral Nerves, March 14-17, 2018, in Orlando, Florida, and awarded the Charles Kuntz Scholarship Award.

\section{Correspondence}

Nader S. Dahdaleh: Northwestern University Feinberg School of Medicine, Chicago, IL. nader.dahdaleh@northwestern.edu. 